\title{
Agonistic autoantibodies: do they have a role in the pathophysiology of SSc?
}

The occurrence of vascular complications is a major determinant of survival in patients with systemic sclerosis (SSc), although the precise mechanisms that underlie the vasculopathy associated with this disease are not fully understood. Angiotensin II and endothelin 1, which both occur at increased levels in patients with SSc, promote vasoconstriction, inflammation and fibrosis, and blockade of their respective receptors (angiotensin II type 1 receptor [ $\mathrm{AT}_{1} \mathrm{R}$ ] and endothelin 1 type $A$ receptor $\left[\mathrm{ET}_{\mathrm{A}} \mathrm{R}\right]$ ) seems to improve some vascular SSc manifestations. Riemekasten et al. examined whether stimulation of these receptors by autoreactive agonistic antibodies might contribute to SSc pathophysiology.

Serum samples were obtained from 478 patients with limited or diffuse SSc, 372 healthy individuals, and 311 patients with 'control' diseases (rheumatoid arthritis, morphea, primary Raynaud phenomenon or primary Sjögren's syndrome). Patients with SSc had significantly higher levels of anti-AT $\mathrm{R}_{1}$ and anti-ET $\mathrm{A}_{\mathrm{A}} \mathrm{R}$ autoantibodies compared with all control groups. Levels of the two autoantibodies correlated strongly ( $r=0.917, P \leq 0.01)$. The antibodies were shown to have biological activity, which could be attenuated by relevant receptor antagonists. High levels of the autoantibodies seemed to be associated with increased SSc severity and an increased risk of SSc-related mortality.

The authors conclude that functional autoimmunity involving $\mathrm{AT}_{1} \mathrm{R}_{\text {and }} \mathrm{ET}_{\mathrm{A}} \mathrm{R}$ is prevalent in patients with SSc, and that the autoantibodies responsible could potentially be used as diagnostic markers in the disease.

Nick Warde

Original article Riemekasten, G. et al. Involvement of functional autoantibodies against vascular receptors in systemic sclerosis. Ann. Rheum. Dis. doi:10.1136/ ard.2010.135772 